\title{
EFECTO INHIBITORIO in vitro DE LA FTALOCIANINA DE ALUMINIO TETRASULFONADA CLORADA FRENTE A Leishmania (Viannia) peruviana Y Leishmania (Viannia) braziliensis
}

\author{
Kelly Vanessa Izarra-Rojas (iD) ${ }^{1, a}$, Nyshon Rojas-Palomino (iD) 2,a, José Luis Gonzales- \\ Medrano $\mathbb{B D}^{4, b}$, Gloria Minaya-Gómez $\mathbb{D}^{2, c}$, Alfredo Berrocal-Huallpa $\mathbb{D}^{3, d}$, \\ Julio Santiago-Contreras (iD) ${ }^{4, e}$, Jorge León-Quispe (iD) ${ }^{1, f}$

\footnotetext{
${ }^{1}$ Laboratorio de Ecología Microbiana, Facultad de Ciencias Biológicas, Universidad Nacional Mayor de San Marcos, Lima, Perú. 2 Laboratorio de Referencia Nacional de Leishmaniasis, Instituto Nacional de Salud, Lima, Perú.

${ }^{3}$ Facultad de Ciencias de la Salud, Universidad Autónoma de Ica, Perú.

${ }^{4}$ Laboratorio de Investigación y Desarrollo de Química Orgánica, Universidad Nacional Mayor de San Marcos, Lima, Perú.

${ }^{a}$ Bióloga/o; ${ }^{b}$ químico; ${ }^{\mathrm{c}}$ bióloga, máster en Medicina Tropical y Salud Internacional, ${ }^{\mathrm{d}}$ biólogo, magister en Mejoramiento Genético de Plantas; ${ }^{\mathrm{e}}$ químico, doctor en Química; ${ }^{\mathrm{f}}$ biólogo, magíster en Ciencias Microbiológicas
}

El presente estudio forma parte de la tesis: Izarra Rojas KV. Actividad fotodinámica in vitro de ftalocianina de aluminio tetrasulfonada clorada (AlPcClS4) frente a estadios extracelular e intracelular de Leishmania (Viannia) peruviana, L. (V.) braziliensis y L. (Leishmania) amazonensis, [Tesis]. Lima: Universidad Nacional Mayor de San Marcos; 2018.

\section{RESUMEN}

Objetivos: Evaluar la actividad fotodinámica in vitro de la ftalocianina de aluminio tetrasulfonada clorada $\left(\mathrm{AlPcClS}_{4}\right)$ sobre promastigotes y amastigotes de Leishmania (Viannia) peruviana y Leishmania (Viannia) braziliensis. Materiales y métodos: La actividad del tratamiento fotodinámico empleando AlPcClS sobre promastigotes y amastigotes de Leishmania fue determinada mediante el método colorimétrico Metil Tiazol Tetrazolium (MTT) y PCR cuantitativo, respectivamente. Resultados: El tratamiento fotodinámico presentó un efecto inhibitorio sobre promastigotes, principalmente sobre Leishmania (V.) peruviana, en menor proporción sobre Leishmania (V.) braziliensis y sobre las formas intracelulares de ambas especies. En Leishmania (V.) peruviana, a las 24 horas posirradiación a $200 \mu \mathrm{M}$ y $350 \mu \mathrm{M}$ el efecto inhibitorio fue del $72,9 \%$ y $73,9 \%$, respectivamente y a las 96 horas fue del $78,8 \%$ y $80,6 \%$, respectivamente. En las formas intracelulares, empleando $200 \mu \mathrm{M}$ y evaluado a las 72 horas postratamiento, se observó una inhibición del $57,8 \%$ de amastigotes de Leishmania (V.) peruviana. $\mathrm{El} \mathrm{IC}_{50}$ fue del 56,5; 50; 44; y 39,7 $\mu \mathrm{M}$, que corresponde a las 24 , 48, 72 y 96 horas posirradiación, respectivamente. Conclusiones: El tratamiento fotodinámico empleando AlPcClS $_{4}$ frente a las especies de Leishmania presentó resultados alentadores principalmente sobre Leishmania (V.) peruviana, lo cual sugiere su potencial uso como alternativa o complemento del tratamiento convencional de la leishmaniasis tegumentaria. Sin embargo, aún se requiere continuar con nuevos ensayos para determinar el índice de selectividad sobre el parásito en su forma intracelular, y desarrollar estrategias que faciliten el ingreso eficiente de la molécula hacia la célula hospedera y al parásito.

Palabras clave: Enfermedades tropicales; Leishmaniasis Cutánea; Terapia Fotodinámica; Fotosensibilizadores; Tratamiento Alternativo (fuente: DeCS BIREME).

Citar como: Izarra-Rojas KV, RojasPalomino N, Gonzales-Medrano JL, Minaya-Gómez G, Berrocal-Huallpa A, Santiago-Contreras J, et al. Efecto inhibitorio in vitro de la ftalocianina de aluminio tetrasulfonada clorada frente a Leishmania (Viannia) peruviana y Leishmania (Viannia) braziliensis. Rev Peru Med Exp Salud Publica. 2020;37(3):462-70. doi: https://doi.org/10.17843/ rpmesp.2020.373.4465

Correspondencia: Nyshon Rojas Palomino; Laboratorio de Leishmaniasis, Instituto Nacional de Salud, Lima, Perú; nyshrojas@gmail.com

Recibido: 16/04/2019

Aprobado: $17 / 06 / 2020$

En línea: $31 / 07 / 2020$

\section{In vitro INHIBITORY EFFECT OF ALUMINUM PHTHALOCYANINE TETRASULFONATE CHLORIDE AGAINST Leishmania (Viannia) Peruviana AND Leishmania (Viannia) Braziliensis}

\begin{abstract}
Objectives: To evaluate the in vitro photodynamic activity of aluminum phthalocyanine tetrasulfonate chloride (AlPc$\mathrm{ClS}_{4}$ ) on promastigotes and amastigotes of Leishmania (Viannia) peruviana and Leishmania (Viannia) braziliensis. Materials and methods: The activity of photodynamic therapy using $\mathrm{AlPcClS}_{4}$ on Leishmania promastigote and amastigotes was determined by the Methyl Thiazole Tetrazolium (MTT) colorimetric method and quantitative PCR, respectively. Results: Photodynamic treatment showed an inhibitory effect on promastigotes, particularly on Leishmania (V.) peruviana, to a lesser extent on Leishmania (V.) braziliensis and also on intracellular forms of both species. At 24 hours post-radiation, using concentrations of $200 \mu \mathrm{M}$ and $350 \mu \mathrm{M}$, the inhibitory effect on Leishmania (V.) peruviana was $72.9 \%$ and $73.9 \%$ respectively; at 96 hours the inhibitory effect was of $78.8 \%$ and $80.6 \%$, respectively. Regarding intracellular forms, the inhibitory effect on Leishmania $(V$.$) peruviana amastigotes was 57.8 \%$ at 72 hours post-treatment, using a concentration of $200 \mu \mathrm{M}$. The $\mathrm{IC}_{50}$ was $56.5,50,44$ and $39.7 \mu \mathrm{M}$, at $24,48,72$ and 96 hours post-radiation, respectively. Conclusions: Photodynamic therapy using AlPcClS4 against Leishmania species showed encouraging results, mainly on Leishmania (V.) peruviana, suggesting a potential use as an alternative or complement to the usual treatment of tegumentary leishmaniasis. However, new trials are still required to determine the selectivity index for the intracellular form of the parasite, and to develop methods to facilitate the efficient entry of the molecule into the host cell and the parasite.
\end{abstract}

Keywords: Tropical Diseases; Cutaneous Leishmaniasis; Photodynamic Therapy Photosensitizers; Alternative Treatment (source: MeSH NLM). 


\section{INTRODUCCIÓN}

La leishmaniasis es una enfermedad metaxénica causada por un protozoario del género Leishmania; en las Américas, es transmitido por un flebotomino del género Lutzomyia conocido como «manta blanca» o «titira» a mamíferos, entre ellos, el ser humano ${ }^{(1)}$. Según la especie de Leishmania infectante y el sistema inmune del hospedero, esta enfermedad puede, además de presentar lesiones ulcerativas, desarrollar metástasis en las mucosas e incluso causar daño en el hígado, bazo y páncreas, que al no tratarse oportunamente pueden producir la muerte del paciente ${ }^{(2)}$.

En el Perú, el Ministerio de Salud reportó en el 2018 un total de 5808 casos de leishmaniasis tegumentaria, de los cuales el $94 \%$ y $6 \%$ representan casos cutáneos y cutáneos mucosos, respectivamente, causados principalmente por Leishmania (Viannia) peruviana, Leishmania (V.) braziliensis, Leishmania (V.) guyanensis y Leishmania (Leishmania) amazonensis; otras formas clínicas inusuales reportadas en nuestro país son la leishmaniasis cutánea diseminada causada por especies como Leishmania (Viannia) braziliensis, Leishmania (V.) guyanensis, Leishmania (V.) peruviana y Leishmania (L.) amazonensis y la leishmaniasis cutánea difusa causada por la infección con Leishmania (L.) amazonensis; por otro lado, en el Perú no hay reportes de casos con leishmaniasis visceral, cuya forma clínica es letal si no es detectada oportunamente.

El tratamiento de primera línea para todas las especies de Leishmania son los antimoniales pentavalentes, mientras que el tratamiento con anfotericina B se corresponde con la segunda línea; ambos son administrados por vía parenteral en prolongadas sesiones. Debido a la naturaleza de estos medicamentos, su empleo provoca efectos adversos, como náuseas, vómitos, mialgias, efectos nefrotóxicos, entre otros, que podrían generar casos de abandono al tratamiento e incrementar el reporte de fallas terapéuticas debido a la administración de dosis incompletas o inadecuadas, además su empleo no es recomendado en mujeres embarazadas ni en pacientes con arritmias cardiacas ${ }^{(3-5)}$. Asimismo, la miltefosina empleada como tratamiento por vía oral y recomendado por la Organización Mundial de la Salud también presenta efectos colaterales, además de elevar el costo del tratamiento de la enfermedad ${ }^{(5,6)}$. Estas limitaciones han estimulado la búsqueda de alternativas terapéuticas más eficaces y con menos efectos colaterales.

La terapia fotodinámica (TFD) ha demostrado que puede inducir la muerte celular por apoptosis y necrosis mediada por el oxígeno singulete y radicales tóxicos (especies reactivas de oxígeno) de alta reactividad producidos por la interacción del agente fotosensibilizador, una longitud de onda específica y oxígeno molecular ${ }^{(7)}$, características que lo convierten en una potencial alternativa para el tratamiento de enfermedades infecciosas, entre ellas, la leishmaniasis ${ }^{(8-10)}$. El presente

\section{MENSAJES CLAVE}

Motivación para realizar el estudio: Los antimoniales pentavalentes usados en el tratamiento de la leishmaniasis ocasionan fallas terapéuticas por la administración de dosis incompletas o inadecuadas, o por abandono del tratamiento, por lo que es necesario buscar nuevos agentes terapéuticos menos tóxicos que sustituyan o complementen el tratamiento actual.

Principales hallazgos: En un modelo in vitro se determinó que el fotosensibilizador ftalocianina de aluminio tetrasulfonada clorada $\left(\mathrm{AlPcClS}_{4}\right)$ inhibe el crecimiento de promastigotes y amastigotes de Leishmania (V.) peruviana y Leishmania (V.) braziliensis.

Implicancias: La capacidad inhibitoria del tratamiento fotodinámico con AlPcClS4 contra la Leishmania motiva a continuar con estudios para mejorar el índice de selectividad mediante nuevas formulaciones y evaluar el vector adecuado que permita la mayor absorción en modelos in vivo.

estudio tiene el objetivo de evaluar in vitro el efecto de un nuevo fotosensibilizador frente a los estadios de promastigotes y amastigotes de Leishmania (V.) peruviana y Leishmania (V.) braziliensis, especies de importancia epidemiológica en la salud pública del Perú.

\section{MATERIALES Y MÉTODOS}

El presente estudio de diseño descriptivo observacional se desarrolló en los laboratorios de Ecología Microbiana (Facultad de Ciencias Biológicas) y de Química Orgánica (Facultad de Química) de la Universidad Nacional Mayor de San Marcos, y en el Laboratorio de Referencia Nacional de Leishmaniasis del Instituto Nacional de Salud (INS) del Perú.

\section{Línea celular macrófagos y promastigotes}

Los ensayos en líneas celulares se desarrollaron en placas de 24 pocillos (Corning, Costar Cat. COR-3524), a los que se le agregó una laminilla circular en la base. La línea celular de macrófago de canes DH82 fue proporcionada por el Laboratorio de Cultivo Celular del INS, cultivada en un medio mínimo esencial (MEM) (Gibco Cat. 61100-061), suplementada con suero fetal bovino inactivado (SFBi) (Gibco, Cat. 1079255) al 15\% y antibióticos (Gibco, Cat. 15240-062), incubada a $37^{\circ} \mathrm{C}, 5 \%$ de $\mathrm{CO}_{2}$ y mantenida por repiques sucesivos cada 72 horas.

Asimismo, se emplearon cultivos de cepas de Leishmania (V.) peruviana y Leishmania (V.) braziliensis, criopreservados a - $70 \pm 5{ }^{\circ} \mathrm{C}$. Para su reactivación, las cepas de Leishmania fueron descongeladas e inmediatamente llevadas a un medio bifásico en agar sangre con $15 \%$ de sangre de conejo, luego a medio líquido Schneider's Drosophila a un pH de 6,65-6,75 
(Gibco, Cat. 21720024) suplementado al 20\% con SFBi ${ }^{(1,12),}$ más $150 \mu \mathrm{g} / \mathrm{ml}$ de gentamicina e incubadas a $24 \pm 1{ }^{\circ} \mathrm{C}$, con la finalidad de obtener mayor masa parasitaria. Las cepas reactivadas se mantuvieron en medio Schneider's al 10\% con SFBi. El proceso de infección in vitro en macrófagos se desarrolló con promastigotes metacíclicos obtenidos por inducción a un pH ácido de 5,5 con $10 \%$ de SFBi y antibióticos.

El proceso de infección de macrófagos tuvo lugar en placas de 24 pocillos de $1 \times 10^{5}$ macrófagos $/ \mathrm{ml}$, en MEM con SFBi al $15 \%$, e incubados a $37^{\circ} \mathrm{C}$ y $5 \%$ de $\mathrm{CO}_{2}$ por 15 horas. Se agregaron promastigotes metacíclicos en una proporción 20:1 (parásito:macrófago) y se incubaron a $33^{\circ} \mathrm{C}$ y $5 \%$ de $\mathrm{CO}_{2}$ por 5 horas. Finalmente, se realizaron tres lavados con MEM al $2 \%$ y con SFBi para eliminar los parásitos no fagocitados.

\section{Evaluación de la actividad inhibitoria}

Se preparó una solución del fotosensibilizador ftalocianina de aluminio tetrasulfonada clorada $\left(\mathrm{AlPcClS}_{4}\right)$ (Frontier Scientic) a una concentración inicial de $1 \mathrm{mM}$ más $0,5 \%$ de dimetilsulfóxido (DMSO) (Applichem) en solución tampón fosfato salino (PBS) a un $\mathrm{pH}$ de 7,4 y filtrado a 0,45 $\mu \mathrm{m}$.

Para determinar la concentración inhibitoria media $\left(\mathrm{IC}_{50}\right)$ de la $\mathrm{AlPcClS}_{4}$, se desarrollaron dos ensayos independientes , cada uno de ellos por triplicado; en promastigotes, se evaluaron a las concentraciones de $0 ; 25 ; 50 ; 75 ; 100 ; 200$ y $350 \mu \mathrm{M}$ del compuesto en $3 \mathrm{ml}$ de medio líquido con $5 \times 10^{5}$ parásitos $\left(\mathrm{T}_{0}\right)$ e incubados durante 24 horas a $26^{\circ} \mathrm{C}$ y en oscuridad. $\mathrm{Al}$ término, se irradió por única vez luz LED a longitud de onda específica de $675 \mathrm{~nm}, 30 \mathrm{~J} / \mathrm{s}^{\star} \mathrm{m}^{2}$ de potencia y a una distancia de $10 \mathrm{~cm}$ por 30 minutos, el cual permitió una potencia de irradiación aproximada de $5,4 \mathrm{~J} / \mathrm{cm}^{2}$. Asimismo, el efecto inhibitorio de la TFD con ftalocianina sobre los promastigotes de Leishmania a las 0, 24, 48, 72 y 96 horas fue determinado indirectamente mediante el ensayo colorimétrico de MTT (Metil Tiazol Tetrazolium) ${ }^{(13)}$.

En amastigotes intracelulares, luego del proceso de infección y lavado, se adicionó $1 \mathrm{ml}$ de MEM con SFBi al 15\% y la $\mathrm{AlPcClS}_{4}$ a una concentración final de 50, 75, 100 y $200 \mu \mathrm{M}$, se incubaron las muestras por 24 horas a $37^{\circ} \mathrm{C}$ y $5 \%$ de $\mathrm{CO}_{2}$. $\mathrm{Al}$ término se irradió con luz LED y volvieron a incubarse por 72 horas posirradiación, el efecto del compuesto sobre las formas amastigotes fue determinado por PCR cuantitativo.

Se evaluó de manera independiente el efecto de la AlPc$\mathrm{ClS}_{4}$ sin exposición a la luz y viceversa. Asimismo, se emplearon promastigotes y macrófagos DH82 como control de crecimiento y estibogluconato (Pentostam) a concentraciones de 100,200 y $2 \times 10^{4} \mu \mathrm{M}$ para los ensayos con promastigotes y a $200 \mu \mathrm{M}$ para los amastigotes intracelulares, además se usó tritón al 0,01\% como control de tratamiento.

\section{Ensayo colorimétrico de MTT}

El método colorimétrico de MTT se desarrolló según la metodología de descrita por Mesa et al. ${ }^{(14)}$ con las siguientes modificaciones: se colectaron en tubos cónicos estériles de $1,5 \mathrm{ml}$ los promastigotes tratados a distintas concentraciones de ftalocianina e irradiados; los parásitos fueron centrifugados a $3500 \mathrm{rpm}$; se descartó cuidadamente el sobrenadante; luego se agregó $450 \mu \mathrm{l}$ de medio líquido y $50 \mu \mathrm{l}$ de MTT $(5 \mathrm{mg} / \mathrm{ml})$, y se incubaron por 4 horas a $32^{\circ} \mathrm{C}$. Finalmente, para detener la formación de los cristales de formazán se agregó a los pocillos una solución de dodecilsulfato de sodio (SDS) al 10\%, $\mathrm{HCl} 0,01 \mathrm{~N}^{(14)}$. Las muestras se leyeron a una absorbancia de $570 \mathrm{~nm}$ en el espectrofotómetro de microplacas Eon (Biotek).

\section{Extracción de ADN y cuantificación de la carga parasitaria}

Los macrófagos infectados y tratados, 72 horas posirradiación, fueron lavados con PBS estéril a un $\mathrm{pH}$ de 7,2 para retirar remanente del medio de cultivo y extracción del ADN. Se agregó $100 \mu \mathrm{l}$ de tripsina-EDTA al pocillo y se incubó por 10 minutos para desprender las células de las laminillas. Luego se adicionó $100 \mu \mathrm{l}$ de PBS, se colectó la suspensión de células y se puso en tubos cónicos de 1,5 $\mathrm{ml}$ para extraer ácidos nucleicos, lo cual se realizó empleando PureLink ${ }^{\circledR}$ Genomic DNA Kit (Invitrogen, Cat. K1820-02), las muestras fueron eluídas en volumen final de $60 \mu \mathrm{l}$.

La medición del efecto inhibitorio del tratamiento fotodinámico en macrófagos infectados fue realizado mediante la cuantificación de la carga parasitaria por $\mathrm{qPCR}{ }^{(15,16)}$. Se emplearon los oligonucleótidos Leis.L1 5'-GACGCACTAAACCCCTCCAA-3' y Leis.U1 5'-AAGTGCTTTCCCATCGCAACT-3' como iniciadores del PCR, y Leis.P1 FAM 5'-CGGTTCGGTGTGTGGCGCC-3' TAMRA como sonda marcada; la metodología se describe en Wortmann et al. ${ }^{(15)}$.

Brevemente, se elaboró la curva estándar con $\mathrm{ADN}$ genómico de Leishmania (V.) braziliensis a una concentración de 415,75 ng equivalente a $5 \times 10^{6}$ parásitos/ $\mu$ l (120 parásitos $=10 \mathrm{pg}$ de $\mathrm{ADN}$ ) y diluciones equivalentes a $10^{6}, 10^{5}, 10^{4}$, $10^{3}, 10^{2}, 10,10^{-1}$ parásitos El volumen final de la reacción fue de $20 \mu \mathrm{l}$, constituido $1 \mathrm{X}$ de Kapa Probe Fast qPCR Master Mix (Kapa Biosystems, Cat. KK4701), 0,2 $\mu \mathrm{mol} / \mu \mathrm{l}$ de cada cebador, $0,04 \mu \mathrm{mol} / \mu \mathrm{l}$ de sonda y $5 \mu \mathrm{l}$ de $\mathrm{ADN}$ de las muestras. Se amplificó el ADN mediante una denaturación inicial de $95{ }^{\circ} \mathrm{C}$ por 30 segundos para activar la enzima, seguido de 40 repeticiones de denaturación a $95{ }^{\circ} \mathrm{C}$ por 30 segundos y una hibridización/extensión a $60^{\circ} \mathrm{C}$ por 30 segundos.

\section{Análisis estadístico}

Se determinó la media y la desviación estándar de los resultados con Microsoft Excel 2010. Asimismo, se calculó el porcentaje de inhibición a partir de las densidades ópticas encontradas en el ensayo de MTT, empleando la fórmula ${ }^{(17)}$ :

Inhibición de crecimiento $(\%)=\frac{\left(\mathrm{DO}_{570} \text { muestra control }-\mathrm{DO}_{570} \text { muestra } \text { Tto }_{t}\right)}{\left(\mathrm{DO}_{570} \mathrm{~nm} \text { muestra control }\right)} \times 100$

Dónde DO = densidad óptica $570 \mathrm{~nm}$ 
Adicionalmente, se realizó el gráfico de QQ plot y la prueba de Shapiro Wilk para evaluar la normalidad y la homogeneidad de los valores residuales de los datos empleando el gráfico de dispersión. Se compararon las medias de la TFD según las concentraciones de 0, 25, 50, 75, 100, 200 y $350 \mu \mathrm{M}$ de $\mathrm{AlPcClS}_{4}$ sobre Leishmania (V.) peruviana y Leishmania (V.) braziliensis empleando ANOVA y la prueba de Tukey, con un $\alpha=0,05$. Asimismo, se determinó la $\mathrm{IC}_{50}$ frente a promastigotes de Leishmania.

\section{Criterios éticos}

El presente estudio fue aprobado por el Comité de Ética del Instituto Nacional de Salud del Perú con RD N. ${ }^{\circ}$ 397-2016-OGITT-OPE/INS.

\section{RESULTADOS}

Mediante el ensayo MTT, se cuantificó el efecto de la TFD sobre Leishmania. Para Leishmania (V.) peruviana, el tratamiento control presentó una $\mathrm{DO}_{570}$ de $0,27 \pm 0,04$; para $100 \mu \mathrm{M}$ presentó una $\mathrm{DO}_{570}$ de $0,22 \pm 0,05$, para $200 \mu \mathrm{M}$ presentó una $\mathrm{DO}_{570}$ de $0,15 \pm 0,03$, y para $350 \mu \mathrm{M}$ presentó una $\mathrm{DO}_{570}$ de $0,14 \pm 0,02$ (Figura 1). A las 24 horas posirradiación de $50 \mu \mathrm{M}$ de fotosensibilizador esta especie presentó

Leishmania (V.) peruviana

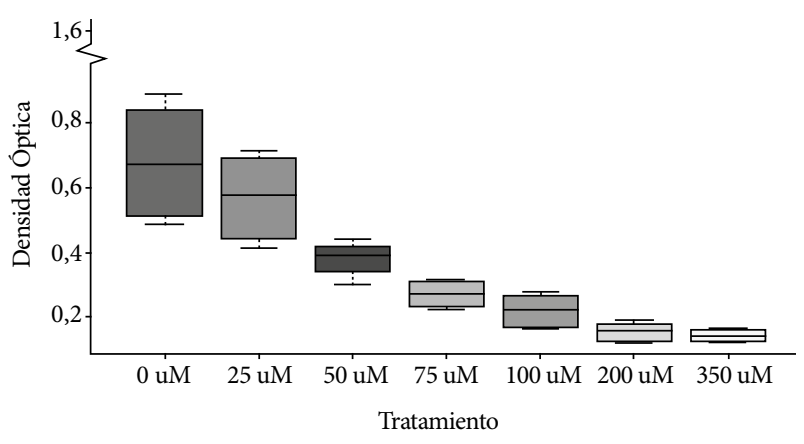

Leishmania (V.) braziliensis

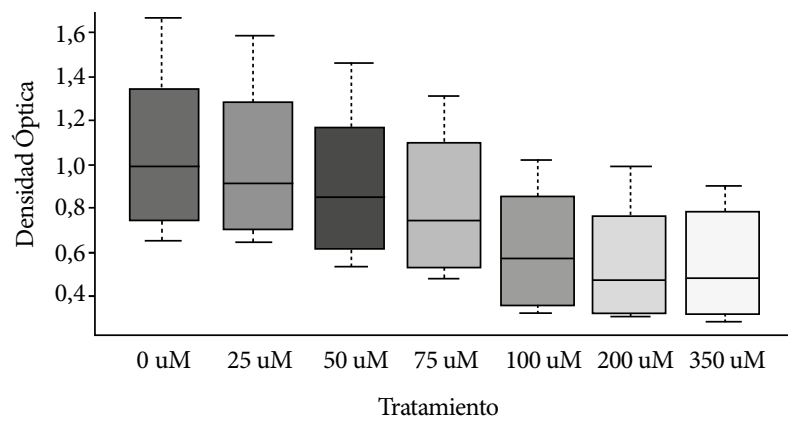

Figura 1. Densidad óptica media y desviación estándar de la cuantificación de los cristales de formazán según el ensayo de MTT (Metil Tiazol Tetrazolium), a diferentes concentraciones de ftalocianina en Leishmania (V.) peruviana y Leishmania (V.) braziliensis una inhibición del 26\% del crecimiento parasitario en comparación a la muestra control; a las concentraciones de 100, 200 y $350 \mu \mathrm{M}$ se presentó una inhibición del crecimiento parasitario del $65,6 \% ; 72,9 \%$ y $73,9 \%$, respectivamente.

A las 96 horas posirradiación el porcentaje de inhibición alcanzó el 68,9\%; 78,8\% y 80,1\%, respectivamente. También se determinaron las $\mathrm{IC}_{50}$ de 56,$5 ; 50 ; 44$; y $39,7 \mu \mathrm{M}$ correspondiente a las 24, 48, 72 y 96 horas, respectivamente (Figura 2). Para la forma intracelular, a las 72 horas posexposición las concentraciones de 50, 75, 100 y $200 \mu \mathrm{M}$ del fotosensibilizador alcanzaron una inhibición de los amastigotes de Leishmania (V.) peruviana de 20,5\%; 46,5\%; 65,2\% y 57,8\%, respectivamente (Tabla 1 ).

Los valores del efecto de la TFD sobre los promastigotes de Leishmania fueron analizados independientemente para cada especie; cada ensayo estuvo conformado por 4 tiempos de evaluación realizados por triplicado a 7 concentraciones diferentes, se analizó un total de 28 tratamientos por especie; los datos obtenidos para Leishmania (V.) peruviana y Leishmania (V.) braziliensis presentaron una distribución normal para ( $\mathrm{p}=0,1041$ y 0,1036 , respectivamente). La comparación de medias por ANOVA permitió determinar que existe diferencia estadísticamente significativa entre los tratamientos en ambas especies de Leishmania $(\mathrm{p}=0,0001)$.

Empleando la prueba de Tukey $(\alpha=0,05)$, se encontró que la TFD en Leishmania (V.) peruviana con 200 y $350 \mu \mathrm{M}$ de $\mathrm{AlPcClS}_{4}$, evaluadas cada una de ellas a 24 y 48 horas, no presentaron diferencias estadísticamente significativas $(p>0,05)$. De manera similar, estas concentraciones evaluadas a las $72 \mathrm{y}$ 96 horas posexposición tampoco presentaron diferencias estadísticamente significativas ( $\mathrm{p}>0,05)$ (Figura 3 ).

El tratamiento solo con irradiación a $670 \mathrm{~nm}$ y a $5,4 \mathrm{~J} / \mathrm{cm}^{2} \mathrm{sin}$ exposición al agente fotosensibilizador y viceversa, no evidenció efecto fototóxico en comparación con el control de crecimiento.

\section{DISCUSIÓN}

El presente estudio evaluó el efecto fototóxico de la ftalocianina de aluminio tetrasulfonada clorada en un ensayo in vitro de fototerapia sobre promastigotes de Leishmania (V.) braziliensis y Leishmania (V.) peruviana, mediante el método MTT, que permitió la cuantificación de los cristales de formazán resultante de la actividad enzimática de la enzima deshidrogenasa mitocondrial de los parásitos vivos ${ }^{(13)}$. El tratamiento fotodinámico consistió en la irradiación con luz LED a una longitud de onda de $670 \mathrm{~nm}$ y potencia de $5,4 \mathrm{~J} / \mathrm{cm}^{2}$ sobre los promastigotes de Leishmania en medio de cultivo a las concentraciones de 25; 50; 75; 100; 200 y $350 \mu \mathrm{M}$ de AlPcClS $_{4}$, según la metodología descrita por Amin et al. ${ }^{(7)}$ sobre células neoplásicas. En amastigotes, la valoración del efecto de la TFD se desarrolló mediante un PCR cuantitativo.

Las ftalocianinas son compuestos orgánicos químicamente estables que han recibido particular atención en la fototerapia como agente sensibilizador principalmente en 

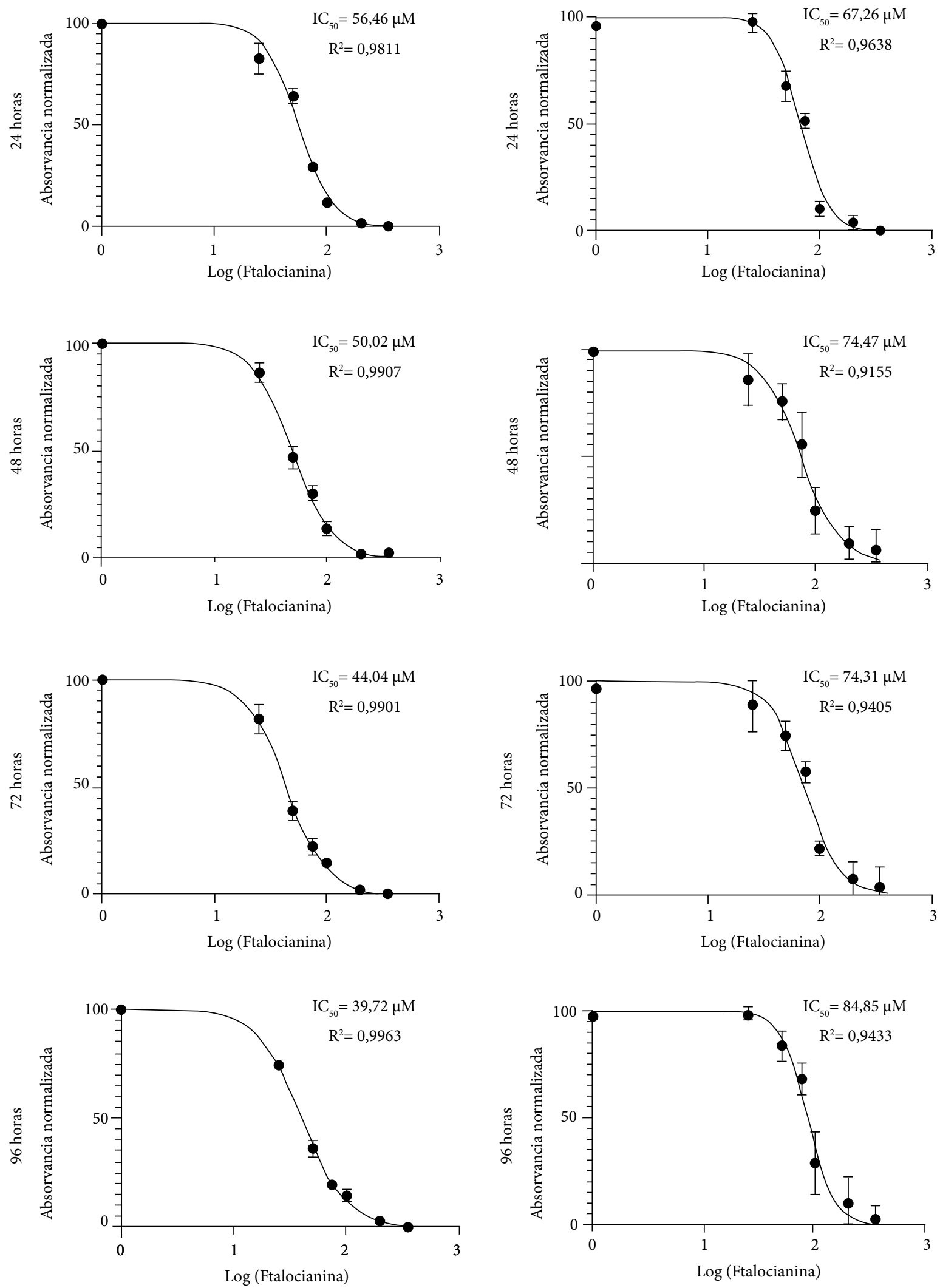

Leishmania (V.) peruviana

Leishmania (V.) braziliensis

Figura 2. Concentración inhibitoria media $\left(\mathrm{IC}_{50}\right)$ de la ftalocianina de aluminio clorada en el tratamiento fotodinámico sobre Leishmania (V.) peruviana y Leishmania (V.) braziliensis, evaluada a las $24,48,72$ y 96 horas posirradiación 
Tabla 1. Determinación de la concentración inhibitoria media $\left(\mathrm{IC}_{50}\right)$ e índice de selectividad de la ftalocianina de aluminio tetrasulfonada clorada y estibogluconato de sodio frente a estadios de promastigotes y amastigotes de Leishmania (V.) peruviana y Leishmania (V.) braziliensis

\begin{tabular}{|c|c|c|c|c|c|c|c|c|c|}
\hline \multirow{2}{*}{ Especie } & \multirow{2}{*}{ Horas } & \multicolumn{6}{|c|}{ \% Inhibición } & \multirow{2}{*}{$\mathrm{IC}_{50}$} & \multirow{2}{*}{ IC 95\% } \\
\hline & & $25 \mu \mathrm{M}$ & $50 \mu \mathrm{M}$ & $75 \mu \mathrm{M}$ & $100 \mu \mathrm{M}$ & $200 \mu \mathrm{M}$ & $350 \mu \mathrm{M}$ & & \\
\hline \multicolumn{10}{|l|}{ Ftalocianina } \\
\hline \multicolumn{10}{|l|}{ Promastigotes } \\
\hline \multirow{4}{*}{ L. (V.) peruviana } & 24 & 12,89 & 26,09 & 52,47 & 65,60 & 72,88 & 73,90 & 56,46 & $53,49-59,36$ \\
\hline & 48 & 10,24 & 40,89 & 53,53 & 66,15 & 75,97 & 76,51 & 50,02 & $48,30-51,75$ \\
\hline & 72 & 14,53 & 48,67 & 61,81 & 67,71 & 78,40 & 79,77 & 44,04 & $42,42-45,69$ \\
\hline & 96 & 20,86 & 51,33 & 64,86 & 68,91 & 78,78 & 80,61 & 39,72 & $38,75-40,70$ \\
\hline \multirow{4}{*}{ L. (V.) braziliensis } & 24 & 0,81 & 16,45 & 25,68 & 49,60 & 53,43 & 55,50 & 67,26 & $63,60-70,94$ \\
\hline & 48 & 5,43 & 14,12 & 26,13 & 44,00 & 52,93 & 54,70 & 74,47 & $67,86-81,47$ \\
\hline & 72 & 2,73 & 11,24 & 19,84 & 37,75 & 44,87 & 47,00 & 74,31 & $69,13-79,66$ \\
\hline & 96 & 3,21 & 10,09 & 17,40 & 34,75 & 42,56 & 44,38 & 75,88 & $71,74-80,07$ \\
\hline \multicolumn{10}{|l|}{ Macrófagos DH82 } \\
\hline L. (V.) peruviana & 72 & ND & 20,52 & 46,45 & 65,16 & 57,78 & ND & 60,28 & $56,78-63,80$ \\
\hline L. (V.) braziliensis & 72 & ND & 4,15 & 9,04 & 20,31 & 36,90 & ND & 94,92 & $90,80-99,63$ \\
\hline \multicolumn{10}{|c|}{ Estibogluconato de sodio } \\
\hline \multicolumn{10}{|l|}{ Promastigotes } \\
\hline \multirow{4}{*}{ L. (V.) peruviana } & 24 & ND & ND & ND & 35,75 & 45,70 & $98,13^{\text {a }}$ & 216,4 & $196,3-240,8$ \\
\hline & 48 & ND & ND & ND & 35,25 & 41,94 & $98,36^{a}$ & 250,1 & $215,4-295,6$ \\
\hline & 72 & ND & ND & ND & 33,25 & 48,58 & $98,43^{\text {a }}$ & 202,1 & $182,7-228,3$ \\
\hline & 96 & ND & ND & ND & 46,45 & 49,04 & $98,35^{\mathrm{a}}$ & 152,3 & $127,4-181,3$ \\
\hline \multirow{4}{*}{ L. (V.) braziliensis } & 24 & ND & ND & ND & 21,40 & 31,73 & $96,88^{a}$ & 389,4 & $325,2-471,2$ \\
\hline & 48 & ND & ND & ND & 21,39 & 30,38 & $96,02^{a}$ & 408,4 & $288,4-604,2$ \\
\hline & 72 & ND & ND & & 27,01 & 37,19 & $96,36^{a}$ & 310 & $257,9-381,3$ \\
\hline & 96 & ND & ND & ND & 30,93 & 33,24 & $97,52^{a}$ & 355,1 & $276,0-470,8$ \\
\hline \multicolumn{10}{|l|}{ Macrófagos DH82 } \\
\hline L. (V.) peruviana & 72 & ND & ND & ND & ND & 54,56 & ND & ND & ND \\
\hline L. (V.) braziliensis & 72 & ND & ND & ND & ND & 50,23 & ND & ND & ND \\
\hline
\end{tabular}

a Evaluado a $2 \times 10^{4} \mu \mathrm{M}$ de antimoniales pentavalentes

$\mathrm{IC}_{50}$ : concentración inhibitoria media; IC95\%: intervalo de confianza al 95\%

ND: no determinado

el tratamiento de enfermedades infecciosas debido a su baja toxicidad y sus propiedades bioquímicas que mejoran la respuesta humoral y celular frente al agente infeccioso o células blanco en caso de neoplasia. Asimismo, participan en la producción de moléculas oxidativas como las especies reactivas de oxígeno y oxígeno singulete, que actúan directamente sobre los residuos aminoacídicos de proteínas y enzimas como cisteína, metionina, triptófano, entre otros, relacionados a la virulencia del patógeno ${ }^{(10)}$. Estudios relacionados con la TFD como alternativa al tratamiento de leishmaniasis evaluaron diversos fotosensibilizadores como la ftalocianina de zinc ( $\mathrm{ZnPc}$ ), de aluminio (AlPc) y de aluminio tetrasulfonado (AlPcS4), entre otros, sobre amastigotes y promastigotes de Leishmania (V.) braziliensis, Leishmania (V.) panamensis, Leishmania (L.) amazonensis, Leishmania (L.) chagasi, Leishmania (L.) major y Leishmania (L.) tropica ${ }^{(8,9,18-20)}$, especies causantes de lesiones cutáneas y cutáneo-mucosas ${ }^{(1)}$, encontrando niveles de $\mathrm{IC}_{50}$ prometedores.

Pinto et al. ${ }^{(9)}$ encontraron que el tratamiento fotodinámico in vitro empleando concentraciones de 1 y $10 \mu \mathrm{M}$ de $\mathrm{AlPcClS}_{4}$ e irradiado a una longitud de $659 \mathrm{~nm}$ sobre promastigotes de Leishmania (V.) braziliensis evaluados al cuarto día posirradiación logró una inhibición aproximada del $42 \%$ y $49 \%$, respectivamente y al noveno día posirradiación, una inhibición del $34 \%$ y 41,3\%, respectivamente. En Leishmania (L.) major evaluado en las mismas condiciones, el efecto inhibitorio al cuarto día posirradiación fue del 50\% en ambas concentraciones, mientras que, al noveno día posirradiación la inhibición fue del 32\% y $41 \%$ correspondiente a las concentraciones de 1 y $10 \mu \mathrm{M}$, respectivamente.

Hernández ${ }^{(21)}$ evaluó la ftalocianina de aluminio clorada $(\mathrm{AlPcCl})$ y la ftalocianina de aluminio clorada disulfonada 


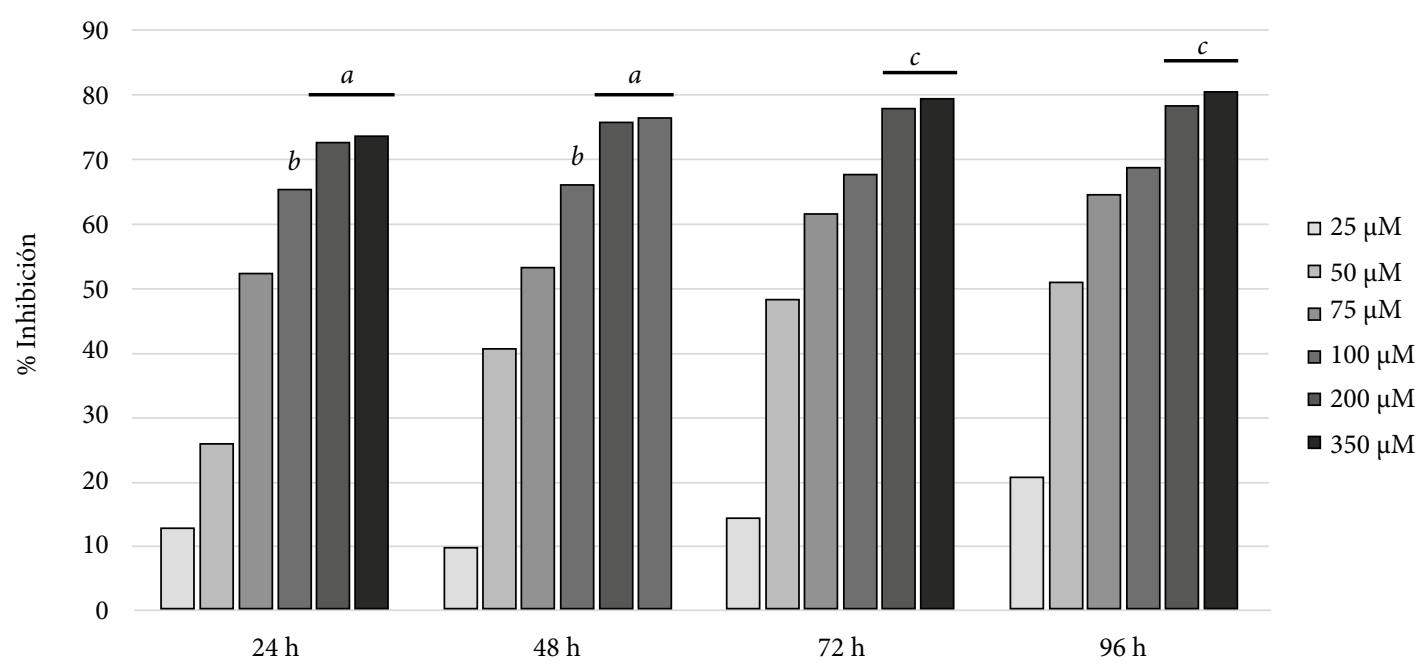

Leishmania (V.) braziliensis

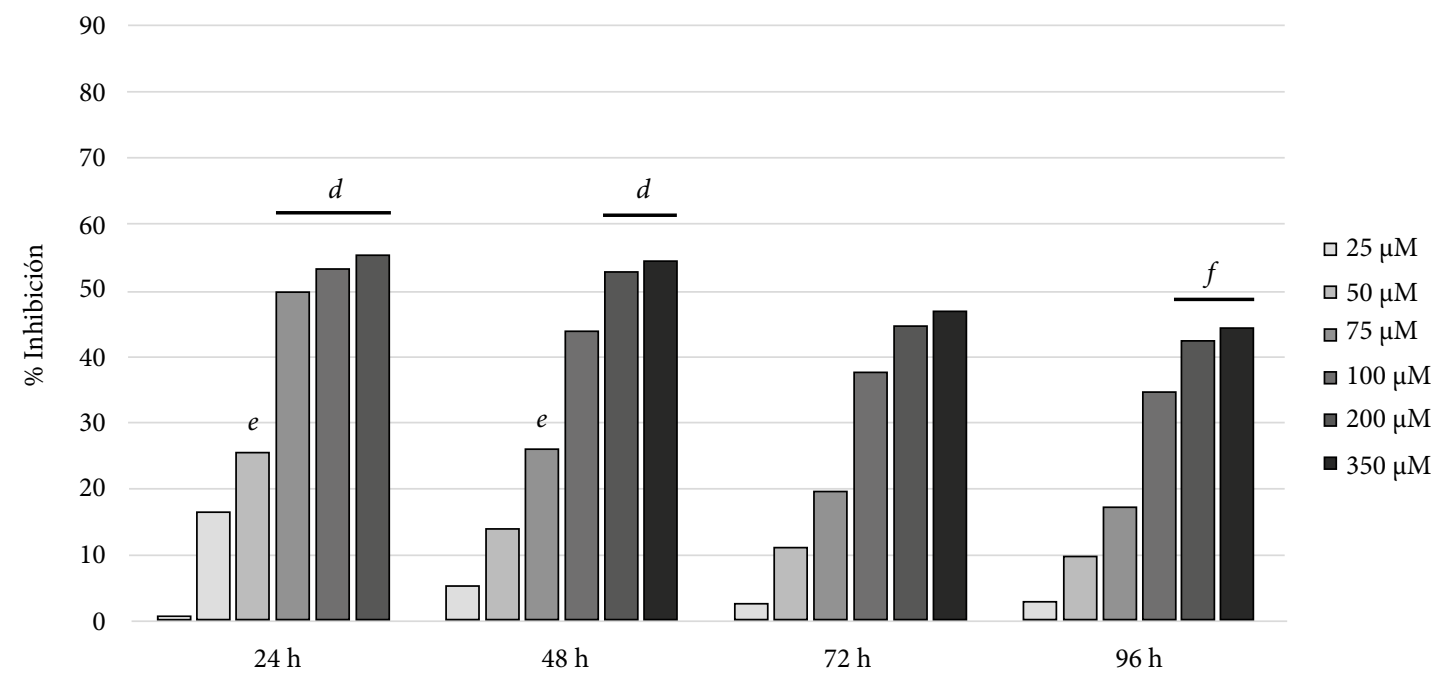

Figura 3. Inhibición del crecimiento parasitario de Leishmania, por exposición al tratamiento fotodinámico con ftalocianina de aluminio tetrasulfonada clorada (a, b, c, d, e y f, representan a tratamientos que no presentan diferencias estadísticamente significativas, prueba de Tukey $\mathrm{p}>0,05$ )

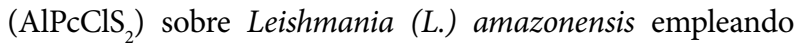
una potencia de irradiación de $3 \mathrm{~J} / \mathrm{cm}^{2}$ y reportó una $\mathrm{IC}_{50}$ de $0,046 \mu \mathrm{M} \pm 0,018$ y una $\mathrm{IC}_{50}$ de $4,101 \mu \mathrm{M} \pm 0,136$, respectivamente. De igual forma Escobar et al. ${ }^{(20)}$ empleando AlPcCl con una irradiación a $10,0 \mathrm{~J} / \mathrm{cm}^{2}$ a una longitud de a $670 \mathrm{~nm}$ reportaron a las 24 horas posirradiación una $\mathrm{IC}_{50}$ de 0,0033 y de $0,17 \mu \mathrm{M}$ en promastigotes de Leishmania (L.) chagasi y de Leishmania (V.) panamensis, respectivamente. Asimismo, la ftalocianina de Zinc ( $\mathrm{ZnPc}$ ) en las mismas condiciones y con las mismas especies evaluadas presentaron una $\mathrm{IC}_{50} \mathrm{de}$ $6,45 \mu \mathrm{M}$ y $6,05 \mu \mathrm{M}$, respectivamente. En nuestro estudio, en el mismo intervalo de tiempo, la $\mathrm{IC}_{50}$ encontrada fue de $56,5 \mu \mathrm{M}$ y de $67,26 \mu \mathrm{M}$ para Leishmania (V.) peruviana y para Leishmania $(V$.$) braziliensis, respectivamente.$
Es probable que los resultados heterogéneos de la TFD se deban al tiempo y fluencia de irradiación, a la longitud de onda empleada, a la naturaleza del compuesto usado como fotosensibilizador (isómeros), a las propiedades fisicoquímicas relacionadas a la internalización celular y a la carga molecular, estas variables junto al rendimiento cuántico del oxígeno singulete, la localización subcelular, el grado de sulfonación, el tamaño de la molécula, la variedad de los iones de metal, la absorción de la luz, la afinidad por el tejido blanco, la selectividad por compartimentos celulares como la mitocondria y la potencia de irradiación alcanzada en las células, podrían influir en la capacidad del compuesto para atravesar la bicapa lipídica de la membrana celular del parásito y reducir los efectos y la eficiencia del tratamiento fotodinámico ${ }^{(22)}$. 
Similar a lo descrito por Amin et al. ${ }^{(7)}$ en el presente estudio la irradiación por sí sola no produjo efecto sobre los promastigotes, macrófagos infectados o macrófagos libres, de igual modo el fotosensibilizador sin exposición a radiación con luz LED no fue tóxico en ninguna de las concentraciones empleadas, lo que es coherente con el principio de la TFD, donde la aplicación independiente del compuesto o irradiación, no generan efecto alguno sobre la viabilidad celular ${ }^{(23)}$.

En el presente estudio no se logró determinar el índice de selectividad (IS), parámetro útil para estimar la efectividad de un compuesto sobre un determinado patógeno ${ }^{(24)}$, tampoco se logró determinar la concentración letal media $\left(\mathrm{DL}_{50}\right)$ sobre la línea celular que contiene la forma intracelular de Leishmania, parámetro importante para la determinación del IS, lo que constituye la principal limitación del estudio.

Asimismo, la TFD podría mejorar el rendimiento del tratamiento convencional, lo que implica diversas posibilidades en el manejo de la leishmaniasis tegumentaria como la reducción de la dosis, el tiempo de aplicación de los tratamientos convencionales, el tratamiento específico por especie, entre otros, y como consecuencia la reducción de sus efectos secundarios ${ }^{(25,26)}$. Sin embargo, aún se requiere desarrollar estudios que determinen el riesgo de reactivación de la enfermedad o recidiva y la respuesta inadecuada al tratamiento; también es importante desarrollar estudios relacionados a evaluar estrategias que permitan mejorar la eficiencia del tratamiento mediante la exposición a más de un ciclo de irradiación. En el presente estudio se empleó una única irradiación con luz LED por 30 minutos, lo cual probablemente no sea suficiente para afectar a la totalidad de los parásitos. Además, es importante desarrollar estudios de investigación relacionados a formular, desarrollar y evaluar el índice de efectividad y selectividad en líneas celulares y modelos animales para estimar la eficiencia del compuesto sobre el parásito.

En conclusión, la $\mathrm{AlPcClS}_{4}$ como fotosensibilizador de la TFD presentó un efecto anti-Leishmania constante principalmente sobre promastigotes de Leishmania (V.) peruviana y, en menor proporción en Leishmania (V.) braziliensis, esta última inició un proceso de recuperación a partir de las 72 horas postratamiento, a diferencia de Leishmania (V.) peruviana que denotó una mayor sensibilidad al tratamiento, sin un proceso de recuperación hasta las 96 horas postratamiento. En las formas de amastigotes el efecto fototóxico fue menor, aun así, la respuesta de los macrófagos DH82 infectados con Leishmania (V.) peruviana fue mayor en comparación a los macrófagos infectados con Leishmania (V.) braziliensis.

Agradecimientos: Al Programa Nacional de Innovación para la Competitividad y Productividad - INNOVATE-PERU (Convenio N.o 177-PNICP-PIAP-2015) por el financiamiento del presente trabajo de investigación. Al Centro Nacional de Salud Pública del Instituto Nacional de Salud, por las facilidades prestadas.

Contribuciones de autoría: KVIR, NRP, JLGM y ABH participaron en la concepción, el diseño de la investigación, la recolección de datos, los resultados, el análisis, la interpretación y la redacción del artículo. NRP, GMG, ABH, JSC y JLQ participaron en la concepción, el diseño, la dirección del trabajo, la revisión crítica del artículo, la aprobación de la versión final, el aporte de materiales de estudio. JSC y JLQ participaron en la obtención financiamiento.

Fuente de financiamiento: El estudio ha sido financiado por el Programa Nacional de Innovación para la Competitividad y Productividad - INNOVATE-PERU (Convenio N.o 177-PNICPPIAP-2015) del Ministerio de la Producción, Lima, Perú.

Conflictos de interés: Los autores declaran no tener conflictos de interés.

\section{REFERENCIAS BIBLIOGRÁFICAS}

1. Ministerio de Salud. Leishmaniasis. Módulos Técnicos. Serie de Documentos Monográficos [Internet]. Lima: MINSA; 2000. 83 p. Disponible en: http://bvs.minsa.gob.pe/local/OGEI/795_MS-OGE106.pdf.

2. Alvar J, Vélez ID, Bern C, Herrero M, Desjeux P, Cano J, et al. Leishmaniasis worldwide and global estimates of its incidence. PLoS One. 2012;7(5):e35671. doi: 10.1371/journal.pone.0035671.

3. Murray HW. Leishmaniasis in the United States: Treatment in 2012. Am J Trop Med Hyg. 2012;86(3):434-40. doi: 10.4269/ajtmh.2012.11-0682

4. Kedzierski L. Leishmaniasis vaccine: Where are we today? J Glob Infect Dis. 2010;2(2):177-85. doi: 10.4103/0974-777X.62881

5. De Menezes JPB, Guedes CES, De Oliveira Almeida Petersen AL, Fraga DBM, Veras PST. Advances in development of new treatment for leishmaniasis. Biomed Res Int. 2015;2015:815023. doi: 10.1155/2015/815023.

6. Soto J, Soto P. Miltefosina oral para el tratamiento de la leishmaniasis. Biomedica. 2006;26 Suppl 1:207-17. doi: 10.7705/biomedica.v26i1.1514.

7. Amin RM, Hauser C, Kinzler I, Rueck A, Scalf-Happ C. Evaluation of photodynamic treatment using aluminum phthalocyanine tetrasulfonate chloride as a photosensitizer: New approach. Photochem Photobiol Sci. 2012;11(7):1156-63. doi: 10.1039/c2pp05411f.
8. Dutta S, Kolli BK, Tang A, Sassa S, Chang KP. Transgenic Leishmania model for delta-aminolevulinate-inducible monospecific uroporphyria: Cytolytic phototoxicity initiated by singlet oxygen-mediated inactivation of proteins and its ablation by endosomal mobilization of cytosolic uroporphyrin. Eukaryot Cell. 2008;7(7):1146-57. doi: 10.1128/EC.00365-07.

9. Pinto JG, Soares CP, Mittmann J. Assessment of Leishmania major and Leishmania braziliensis promastigote viability after photodynamic treatment with aluminum phthalocyanine tetrasulfonate (AlPcS4). J Venom Anim Toxins Incl Trop Dis. 2011;17(3). doi: 10.1590/S167891992011000300010.

10. Dai T, Huang YY, Hamblin MR. Photodynamic therapy for localized infections-State of the art. Photodiagnosis Photodyn Ther. 2009;6(3-4):170-88. doi: 10.1016/j.pdpdt.2009.10.008.

11. Ponte-Sucre A, Díaz E, Padrón-Nieves M. Drug Resistance in Leishmania Parasites [Internet]. Viena: Springer Verlag Wien; 2013 [citado el 12 de abril de 2019]. Disponible en: https://link.springer.com/ book/10.1007/978-3-7091-1125-3\#toc

12. Gamboa D, Torres K, De Doncker S, Zimic M, Arévalo J, Dujardin J-C. Evaluation of an in vitro and in vivo model for experimental infection with Leishmania (Viannia) braziliensis and L. (V.) peruviana. Parasitology. 2008;135(3):319-26. doi: 10.1017/S0031182007003848. 
13. Brito S, Crescente O, Fernández A, Coronado A, Rodriguez N. Eficacia de un ácido kaurénico extraído de la planta venezolana Wedelia trilobata (Asterácea ) contra Leishmania (Viannia) braziliensis. Biomedica. 2006; 26 Suppl 1:180-7. doi: 10.7705/biomedica.v26i1.1511.

14. Mesa AM, Molano PA, Seon B, Figadere B, Robledo SM, Muñoz DL, et al. Síntesis y actividades Leishmanicida y citotóxica in vitro de análogos 2-Arilquinolonas. Rev la Fac Química Farm [Internet]. 2008 [citado el 12 de abril de 2019];15(2):259-66. Disponible en: http://www.scielo. org.co/pdf/vitae/v15n2/v15n2a08.pdf.

15. Wortmann G, Sweeney C, Houng HS, Aronson N, Stiteler J, Jackson $\mathrm{J}$, et al. Rapid diagnosis of Leishmaniasis by fluorogenic polymerase chain reaction. Am J Trop Med Hyg. 2001;65(5). doi: 10.4269/ajtmh.2001.65.583.

16. Gomes LI, Gonzaga FM, Morais-Teixeira E de, de Souza-Lima BS, Freire VV, Rabello A. Validation of quantitative real-time PCR for the in vitro assessment of antileishmanial drug activity. Exp Parasitol. 2012;131(2):175-9. doi: 10.1016/j.exppara.2012.03.021.

17. George SA, Bhadran S, Sudhakar M, Harini BP. Comprehensive in vitro evaluation of pharmacological activities of selected mass spectrometry profiling of flacourtia jangomas flower extract. Asian J Pharm Clin Res. 2017 [citado el 12 de abril de 2019];10(5):237-44. Disponible en: https://innovareacademics.in/journals/index.php/ajpcr/article/ view/17419/10818.

18. Peloi LS, Biondo CEG, Kimura E, Politi MJ, Lonardoni MVC, Aristides SMA, et al. Photodynamic therapy for American cutaneous leishmaniasis: The efficacy of methylene blue in hamsters experimentally infected with Leishmania (Leishmania) amazonensis. Exp Parasitol [Internet]. 2011;128(4). doi: 10.1016/j.exppara.2011.04.009.

19. Fink C, Toberer F, Enk A, Gholam P. Effective treatment of cutaneous leishmaniasis caused by Leishmania tropica with topical photodynamic therapy. JDDG. 2016;14(8). doi: 10.1111/ddg.13082.

20. Escobar P, Hernández IP, Rueda CM, Martínez F, Páez E. Photodynamic activity of aluminium (III) and zinc (II) phthalocyanines in Leishmania promastigotes. Biomedica. 2006 [citado el 27 de junio de
2017];26 Suppl 1(Iii):49-56. Disponible en: http://www.ncbi.nlm.nih. gov/pubmed/17361841.

21. Hernández Peñaranda IP. Actividad fototóxica in vitro e in vivo de ftalocianina de aluminio clorada contra Leishmania amazonensis [Tesis]. Bucaramanga: Universidad Industrial de Santander; 2010.

22. Castano AP, Demidova TN, Hamblin MR. Mechanisms in photodynamic therapy: Part one - Photosensitizers, photochemistry and cellular localization. Photodiagnosis Photodyn Ther. 2004 [citado el 6 de marzo de 2019];1(4):279-93. Disponible en: https://www.ncbi.nlm.nih.gov/pmc/ articles/PMC4108220.

23. Aureliano DP, Ribeiro MS, Lauretta Lindoso JA, Pogliani FC, Sellera FP, Song D, et al. Treatment and Control of Leishmaniasis Using Photodynamic Therapy. En: Leishmaniasis - Trends in Epidemiology, Diagnosis and Treatment [Internet]. 2014 [citado el 17 de abril de 2019]. p. 393-412. Disponible en: https://www.intechopen.com/books/ leishmaniasis-trends-in-epidemiology-diagnosis-and-treatment/treatment-and-control-of-leishmaniasis-using-photodynamic-therapy.

24. Sánchez-Suárez J, Albarracín D, Rojas M, Rincón J, Delgado G. Evaluación de la actividad citotóxica y leishmanicida de extractos y fracciones de Piper cumanense y Piper holtonii Resumen Evaluation of the cytotoxic and leishmanicidal activity of extracts Introducción Rev Colomb Cienc Quím Farm. 2010 [citado el 20 de marzo de 2019] 39. Disponible en: https:// revistas.unal.edu.co/index.php/rccquifa/article/view/22998.

25. Ribeiro JBP, Miranda-Vilela AL, Amorim AAS, Garcia RD, Moreira JR, Gomes CM, et al. Study of the efficacy of N-methyl glucamine antimoniate (SbV) associated with photodynamic therapy using liposomal chloroaluminium phthalocyanine in the treatment of cutaneous leishmaniasis caused by Leishmania (L.) amazonensis in C57BL6 mice. Photodiagnosis Photodyn Ther. 2019;26:261-9. doi: 10.1016/j.pdpdt.2019.04.004.

26. Ribeiro JBP, Miranda-Vilela AL, Graziani D, Gomes MR de A, Amorim AAS, Garcia RD, et al. Evaluation of the efficacy of systemic miltefosine associated with photodynamic therapy with liposomal chloroaluminium phthalocyanine in the treatment of cutaneous leishmaniasis caused by Leishmania (L.) amazonensis in C57BL/6 mice. Photodiagnosis Photodyn Ther. 2016;13:282-90. doi: 10.1016/j.pdpdt.2015.08.006. 